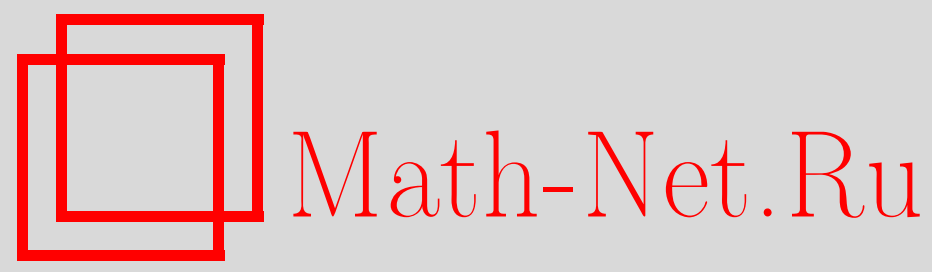

А. В. Псху, О вещественных нулях функции типа МиттагЛеффлера, Матем. заметки, 2005, том 77, выпуск 4, 592599

DOI: https://doi.org/10.4213/mzm2520

Использование Общероссийского математического портала Math-Net.Ru подразумевает, что вы прочитали и согласны с пользовательским соглашением http://www . mathnet.ru/rus/agreement

Параметры загрузки:

IP : 54.197 .217 .227

26 апреля 2023 г., 12:00:25

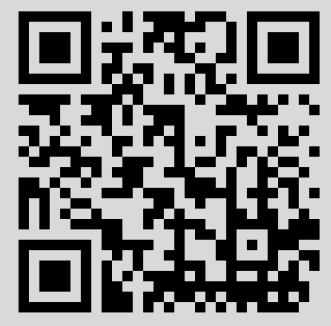




\section{О ВЕЩЕСТВЕННЫХ НУЛЯХ}

\section{ФУНКЦИИ ТИПА МИТТАГ-ЛЕФФЛЕРА}

\section{A. В. Псxy}

В данной заметке доказывается утверждение, позволяющее распространять результаты, связанные с наличием или отсутствием у функций типа Миттаг-Леффлера

$$
E_{1 / \alpha}(z ; \mu)=\sum_{k=0}^{\infty} \frac{z^{k}}{\Gamma(\alpha k+\mu)}
$$

вещественных нулей при определенных значениях $\alpha$ и $\mu$ на более обшшрные области изменения этих параметров. Дается геометрическое описание множеств пар $(\alpha, \mu)$, при которых функция $E_{1 / \alpha}(z ; \mu)$ имеет и при которых не имеет вещественных нулей.

Библиография: 10 названий.

1. Распределению нулей функции типа Миттаг-Леффлера

$$
E_{1 / \alpha}(z ; \mu)=\sum_{k=0}^{\infty} \frac{z^{k}}{\Gamma(\alpha k+\mu)}
$$

посвящено значительное количество работ (см., например, [1], [2] и библиографию там). Вопрос о вещественных нулях функции (1) тесно связан с разрешимостью спектральных задач для уравнений дробного порядка (см. [3, гл. 3, 4]).

В данной заметке доказьвается утверждение, позволяющее распространять результаты, связанные с наличием или отсутствием у функций типа Миттаг- Леффлера вешественных нулей при определенных значениях $\alpha$ и $\mu$, на более обширные области изменения этих параметров. Дается геометрическое описание множеств пар $(\alpha, \mu)$, при которых функция (1) имеет и при которых не имеет вешественных нулей.

2. Далее будем считать $\xi>0, \eta>0$. Обозначим через $B$ множество пар $(\xi, \eta)$ таких, что функция $E_{1 / \xi}(t ; \eta)$ принимает как положительные, так и отрищательные значения, т.е., учитывая, что $E_{1 / \xi}(t ; \eta)>0$ при $t \geqslant 0$ :

$$
B=\left\{(\xi, \eta): \xi, \eta>0, \exists t \in \mathbb{R}, E_{1 / \xi}(t ; \eta)<0\right\} .
$$

Через $B^{*}$ обозначим множество пар $(\xi, \eta)$, для которых функция $E_{1 / \xi}(t ; \eta)$ не имеет вещественных нулей:

$$
B^{*}=\left\{(\xi, \eta): \xi, \eta>0, \forall t \in \mathbb{R}, E_{1 / \xi}(t ; \eta)>0\right\} .
$$


Через $B_{0}$ обозначим множество пар $(\xi, \eta)$ таких, что функция $E_{1 / \xi}(t ; \eta)$ имеет вещественные нули, но не принимает отрицательные значения:

$$
B_{0}=\{(\xi, \eta): \xi, \eta>0\} \backslash\left(B \cup B^{*}\right)
$$

Для фиксированньх $\alpha$ и $\mu$ обозначим через $D_{\alpha, \mu}$ множество точек $(\xi, \eta)$, ограниченное лучом прямой, проходящей через точки $(0,0)$ и $(\alpha, \mu)$, выходящим из точки $(\alpha, \mu)$ в направлении возрастания $\xi$ и $\eta$, отрезком с концами в точках $(\alpha, 0)$ и $(\alpha, \mu)$ и лучом, лежащим на оси $O \xi$, выходящим из точки $(\alpha, 0)$ в положительном направлении. Все точки гранищы, за исключением точек, лежащих на оси $O \xi$, и точки $(\alpha, \mu)$, принадлежат множеству $D_{\alpha, \mu}$. То есть

$$
D_{\alpha, \mu}=\left\{(\xi, \eta): \alpha \leqslant \xi, 0<\eta \leqslant \frac{\mu \xi}{\alpha},(\xi, \eta) \neq(\alpha, \mu)\right\}
$$

Через $D_{\alpha, \mu}^{*}$ обозначим множество, ограниченное лучом, выходящим из точки $(\alpha, \mu)$ параллельно оси $O \eta$, отрезком с концами в точках $(0,0)$ и $(\alpha, \mu)$ и положительной частью оси $O \eta$. Все граничные точки, кроме точек оси $O \eta$ и точки $(\alpha, \mu)$, принадлежат множеству $D_{\alpha, \mu}^{*}$. Или

$$
D_{\alpha, \mu}^{*}=\left\{(\xi, \eta): 0<\xi \leqslant \alpha, \eta \geqslant \frac{\mu \xi}{\alpha},(\xi, \eta) \neq(\alpha, \mu)\right\}
$$

Основным результатом этой работы является следующая

Teоpema 1. 1) Пусть $(\alpha, \mu) \in B^{*} \cup B_{0}$. Тогдa $D_{\alpha, \mu}^{*} \subset B^{*}$.

2) Пусть $(\alpha, \mu) \in B \cup B_{0}$. Тогда $D_{\alpha, \mu} \subset B$.

ДокАЗАТЕЛЬСтво. В работе [4] было введено интегральное преобразование

$$
A^{\beta, \delta} v(x) \equiv\left(A^{\beta, \delta} v\right)(x)=\int_{0}^{\infty} v(t) x^{\delta-1} e_{1, \beta}^{1, \delta}\left(-\frac{t}{x^{\beta}}\right) d t, \quad 0<\beta<1 \text {, }
$$

которое является обобщением преобразования Б. Станковича [5]. Здесь

$$
e_{\nu, \gamma}^{\mu, \varepsilon}(z)=\sum_{k=0}^{\infty} \frac{z^{k}}{\Gamma(\nu k+\mu) \Gamma(-\gamma k+\varepsilon)}
$$

- функция типа Райта. В [4] приведено соотношение

$$
A^{\beta, \delta}\left[x^{\mu-1} E_{1 / \alpha}\left(-x^{\alpha} ; \mu\right)\right]=x^{\mu \beta+\delta-1} E_{1 / \alpha \beta}\left(-x^{\alpha \beta} ; \mu \beta+\delta\right), \quad \mu>0 .
$$

Приведем доказательство этого соотношения.

Известно [6] асимптотическое разложение функции Райта при $x \rightarrow \infty$

$$
e_{1, \beta}^{1, \delta}(-x)=Y^{1 / 2-\beta} e^{-Y}\left\{\sum_{m=0}^{M-1} a_{m} Y^{-m}+O\left(Y^{-M}\right)\right\}
$$

где $Y=(1-\beta)\left(\beta^{\beta} x\right)^{1 /(1-\beta)}, a_{m}-$ константы, зависящие от $\beta$ и $\delta$. 
Из (4) видно, что интеграл (2) сходится для любой функции $v(x)$, интегрируемой на любом конечном отрезке положительной полуоси и удовлетворяющей асимптотическим неравенствам

$$
\begin{array}{lll}
|v(x)|<c x^{\varepsilon}, & \varepsilon>-1, \delta \neq 0, \varepsilon>-2, \delta=0, & x \rightarrow 0, \\
|v(x)|<c \exp \left(k x^{\varepsilon}\right), & \varepsilon<\frac{1}{1-\beta}, & x \rightarrow \infty .
\end{array}
$$

Докажем равенство

$$
A^{\beta, \delta} x^{\nu-1}=\frac{\Gamma(\nu)}{\Gamma(\beta \nu+\delta)} x^{\beta \nu+\delta-1} .
$$

Для этого воспользуемся интегральным представлением функции Райта (см. [6], а также [7])

$$
e_{1, \beta}^{1, \delta}(z)=\frac{1}{2 \pi i} \int_{\gamma(\varepsilon, \omega \pi)} e^{p} p^{-\delta} e^{z p^{\beta}} d p
$$

где через $\gamma(\varepsilon, \omega \pi)$ обозначен контур, состоящий из дуги окружности $|p|=\varepsilon>0$, $|\arg p| \leqslant \omega \pi$, и двух лучей $\arg p=\omega \pi$ и $\arg p=-\omega \pi,|p| \geqslant \varepsilon$; значение $\omega$ выбрано так, что выполняются неравенства $1-\omega \beta>1 / 2,1 \geqslant \omega>1 / 2$; контур проходится в направлении неубывания $\arg p$.

Сделаем замену переменной интегрирования $t=s x^{\beta}$, воспользуемся (7) и изменим порядок интегрирования:

$$
\begin{aligned}
A^{\beta, \delta} x^{\nu-1} & =\int_{0}^{\infty} t^{\nu-1} x^{\delta-1} e_{1, \beta}^{1, \delta}\left(-\frac{t}{x^{\beta}}\right) d t=x^{\beta \nu+\delta-1} \int_{0}^{\infty} s^{\nu-1} e_{1, \beta}^{1, \delta}(-s) d s \\
& =\frac{x^{\beta \nu+\delta-1}}{2 \pi i} \int_{\gamma(\varepsilon, \omega \pi)} e^{p} p^{-\delta} \int_{0}^{\infty} t^{\nu-1} e^{-t p^{\beta}} d t d p .
\end{aligned}
$$

Учитьвая, что $\arg \left(p^{\beta}\right)<\pi / 2$, когда $p \in \gamma(\varepsilon, \omega \pi)$ и (см., например, [8, с. 424, 522])

$$
\frac{1}{2 \pi i} \int_{\gamma(\varepsilon, \omega \pi)} e^{p} p^{-\delta-\beta \nu} d p=\frac{1}{\Gamma(\delta+\beta \nu)}, \quad \int_{0}^{\infty} e^{-s} s^{\nu-1} d s=\Gamma(\nu), \quad \nu>0,
$$

получаем (6). Отметим, что в случае $\delta=1-\beta$ соотношение (6) было доказано в [5].

В дальнейшем мы будем использовать асимптотические разложения функции (1). Если $0<\alpha<2$, то при $|z| \rightarrow \infty$ справедливы формулы [9, с. 134]

$$
\begin{aligned}
E_{1 / \alpha}(z ; \mu)= & -\sum_{k=1}^{n} \frac{z^{-k}}{\Gamma(\mu-\alpha k)}+O\left(\frac{1}{|z|^{n+1}}\right), \quad \pi \geqslant|\arg z| \geqslant \frac{\alpha \pi}{2}+\varepsilon, \\
E_{1 / \alpha}(z ; \mu)= & \frac{1}{\alpha} z^{(1-\mu) / \alpha} \exp \left(z^{1 / \alpha}\right) \\
& -\sum_{k=1}^{n} \frac{z^{-k}}{\Gamma(\mu-\alpha k)}+O\left(\frac{1}{|z|^{n+1}}\right), \quad|\arg z| \leqslant \frac{\alpha \pi}{2}+\varepsilon .
\end{aligned}
$$

Если же $\alpha \leqslant 2$, то (см. [9, с. 137])

$$
E_{1 / \alpha}(z ; \mu)=\frac{1}{\alpha} \sum\left(Z_{m}\right)^{1-\mu} \exp \left(Z_{m}\right)-\sum_{k=1}^{n} \frac{z^{-k}}{\Gamma(\mu-\alpha k)}+O\left(\frac{1}{|z|^{n+1}}\right)
$$


где $Z_{m}=z^{1 / \alpha} e^{i 2 \pi m / \alpha}$, а суммирование в первой сумме проводится по тем $m, m=0$, $\pm 1, \pm 2, \ldots$, для которых выполнены неравенства $|\arg z+2 \pi m| \leqslant \alpha \pi / 2, n \in \mathbb{N}$.

Обозначим

$$
E^{n}(x ; \alpha, \mu)=\sum_{k=0}^{n} \frac{(-1)^{k} x^{\alpha k+\mu-1}}{\Gamma(\alpha k+\mu)} .
$$

Из формулы (6) следует равенство

$$
A^{\beta, \delta} E^{n}(x ; \alpha, \mu) \equiv \int_{0}^{\infty} E^{n}(t ; \alpha, \mu) x^{\delta-1} e_{1, \beta}^{1, \delta}\left(-\frac{t}{x^{\beta}}\right) d t=E^{n}(x ; \alpha \beta, \mu \beta+\delta) .
$$

Для правой части (11) имеем

$$
\lim _{n \rightarrow \infty} E^{n}(x ; \alpha \beta, \mu \beta+\delta)=x^{\mu \beta+\delta-1} E_{1 / \alpha \beta}\left(-x^{\alpha} ; \mu \beta+\delta\right) .
$$

Из соотношений (8)-(10) следует, что

$$
\left|E^{n}(x ; \alpha, \mu)\right| \leqslant C \exp (k x),
$$

где $C$ и $k$ - положительные константы, причем, не зависяшие от $n$. С учетом (4) это означает равномерную сходимость несобственного интеграла из (11) на множестве $\{n \in \mathbb{N}\}$. Поэтому можем изменить порядок предельньх переходов:

$$
\lim _{n \rightarrow \infty} A^{\beta, \delta} E^{n}(x ; \alpha, \mu)=\lim _{z \rightarrow+\infty} \lim _{n \rightarrow \infty} \int_{1 / z}^{z} E^{n}(t ; \alpha, \mu) x^{\delta-1} e_{1, \beta}^{1, \delta}\left(-\frac{t}{x^{\beta}}\right) d t .
$$

Учитьвая равномерную сходимость $E^{n}(x ; \alpha, \mu)$ к функции $x^{\mu-1} E_{1 / \alpha}\left(-x^{\alpha} ; \mu\right)$ при $n \rightarrow \infty$ на любом отрезке $[1 / z, z], 1<z<\infty$, получаем

$$
\begin{aligned}
\lim _{n \rightarrow \infty} A^{\beta, \delta} E^{n}(x ; \alpha, \mu) & =\lim _{z \rightarrow+\infty} \int_{1 / z}^{z} \lim _{n \rightarrow \infty} E^{n}(t ; \alpha, \mu) x^{\delta-1} e_{1, \beta}^{1, \delta}\left(-\frac{t}{x^{\beta}}\right) d t \\
& =A^{\beta, \delta} x^{\mu-1} E_{1 / \alpha}\left(-x^{\alpha} ; \mu\right) .
\end{aligned}
$$

Из (11)-(13) следует (3).

Известно [10], что при $\delta \geqslant 0, x>0$,

$$
e_{1, \beta}^{1, \delta}(-x)>0
$$

Поэтому если $u(x) \geqslant 0(u(x) \not \equiv 0, x>0), \delta \geqslant 0$, то

$$
A^{\beta, \delta} u(x)>0 \text {. }
$$

Из (3) и (15) следует, что если $x^{\mu-1} E_{1 / \alpha}\left(-x^{\alpha} ; \mu\right) \geqslant 0, x>0$, то

$$
x^{\mu \beta+\delta-1} E_{1 / \alpha \beta}\left(-x^{\alpha \beta} ; \mu \beta+\delta\right)>0 .
$$

То есть, если $(\alpha, \mu) \in B^{*} \cup B_{0}$, то

$$
(\alpha \beta, \mu \beta+\delta) \in B^{*} \quad \forall \beta \in(0,1), \quad \delta \geqslant 0 .
$$


Справедливо соотношение (см., например, [9, с. 120])

$$
D_{0 x}^{-\varepsilon} x^{\mu-1} E_{1 / \alpha}\left(-x^{\alpha} ; \mu\right)=x^{\mu+\varepsilon-1} E_{1 / \alpha}\left(-x^{\alpha} ; \mu+\varepsilon\right),
$$

где $D_{0 x}^{-\varepsilon}-$ оператор дробного интегрирования в смысле Римана-Лиувилля:

$$
D_{0 x}^{-\varepsilon} u(x)=\frac{1}{\Gamma(\varepsilon)} \int_{0}^{x} \frac{u(t)}{(x-t)^{1-\varepsilon}} d t
$$

Из формулы (17) и положительности ядра интеграла в $(18)$ следует, что если $(\alpha, \mu) \in$ $B^{*} \cup B_{0}$, то

$$
(\alpha, \mu+\varepsilon) \in B^{*} \quad \forall \varepsilon>0 .
$$

Из (16) и (19) следует первое утверждение теоремы.

Докажем второе утверждение. Из (2) и (14) вытекает, что если функция $v(x)=$ $A^{\beta, \delta} u(x)$ обращается в нуль хотя бы в одной точке $x_{0}>0$, то функция $u(x)$ принимает как положительные, так и отрицательные значения. Поэтому из равенства

$$
A^{\beta, \delta}\left[x^{(\mu-\delta) / \beta-1} E_{\beta / \alpha}\left(-x^{\alpha / \beta} ; \frac{\mu-\delta}{\beta}\right)\right]=x^{\mu-1} E_{1 / \alpha}\left(-x^{\alpha} ; \mu\right),
$$

которое эквивалентно (3), следует, что если $(\alpha, \mu) \in B \cup B_{0}$, то

$$
\left(\frac{\alpha}{\beta}, \frac{\mu-\delta}{\beta}\right) \in B \quad \forall \beta \in(0,1), \quad \mu>\delta \geqslant 0 .
$$

На основании равенства

$$
D_{0 x}^{-\varepsilon} x^{\mu-\varepsilon-1} E_{1 / \alpha}\left(-x^{\alpha} ; \mu-\varepsilon\right)=x^{\mu-1} E_{1 / \alpha}\left(-x^{\alpha} ; \mu\right)
$$

получаем, что если $(\alpha, \mu) \in B \cup B_{0}$, то

$$
(\alpha, \mu-\varepsilon) \in B \quad \forall \varepsilon>0, \mu-\varepsilon>0 .
$$

С помощью (20) и (22) приходим ко второму утверждению теоремы. Теорема 1 доказана.

ЗАмЕчанИЕ 1 . Отметим одно следствие формулы $(21)$. Пусть $(\alpha, \mu) \in B \cup B_{0}$. Обозначим через $z(\alpha, \mu)$ наименьший по модулю действительный нуль функции $E_{1 / \alpha}(z ; \mu)$. Очевидно, что $z(\alpha, \mu)<0$. Из $(21)$ следует, что

$$
\left.D_{0 x}^{-\varepsilon} x^{\mu-\varepsilon-1} E_{1 / \alpha}\left(-x^{\alpha} ; \mu-\varepsilon\right)\right|_{x=z_{0}}=0,
$$

где $z_{0}=[-z(\alpha, \mu)]^{1 / \alpha}$. Поэтому существует $z_{1}$ такое, что $z_{1} \in\left(0, z_{0}\right)$ и $E_{1 / \alpha}\left(-z_{1}^{\alpha} ; \mu-\varepsilon\right)$ $=0$. Другими словами,

$$
|z(\alpha, \mu-\varepsilon)|<|z(\alpha, \mu)|
$$

если $\mu>\varepsilon>0$.

3. С помощью теоремы 1 можно доказать следующее утверждение. 
Теорема 2. Справедливы включения

$$
\begin{gathered}
\{(\alpha, \mu): 0<\mu<\alpha\} \subset B, \\
D_{1,1}^{*} \cup D_{2,3}^{*} \subset B^{*}, \quad D_{2,3} \subset B .
\end{gathered}
$$

ДокАЗАТЕЛЬСтво. Пусть $0<\mu<\alpha<2$. Рассмотрим интеграл

$$
\begin{aligned}
\int_{0}^{\infty} x^{\mu-1} E_{1 / \alpha}\left(-x^{\alpha} ; \mu\right) d x & =\lim _{z \rightarrow+\infty} \int_{0}^{z} x^{\mu-1} E_{1 / \alpha}\left(-x^{\alpha} ; \mu\right) d x \\
& =\lim _{z \rightarrow+\infty} z^{\mu} E_{1 / \alpha}\left(-z^{\alpha} ; \mu+1\right) .
\end{aligned}
$$

Учитьвая асимптотическое поведение функции типа Миттаг-Леффлера (8), получаем, что

$$
\int_{0}^{\infty} x^{\mu-1} E_{1 / \alpha}\left(-x^{\alpha} ; \mu\right) d x=0, \quad 0<\mu<\alpha<2 .
$$

Это означает, что функция $E_{1 / \alpha}(x ; \mu)$ принимает как положительные, так и отрицательные значения при $0<\mu<\alpha<2, x \in \mathbb{R}$. Поэтому из теоремы 1 следует (23).

Далее, известно, что

$$
E_{1}(-x ; 1)=e^{-x}, \quad x^{2} E_{1 / 2}\left(-x^{2} ; 3\right)=1-\cos x .
$$

То есть $(1,1) \in B^{*}$, а $(2,3) \in B_{0}$. Из теоремы 1 получаем $(24)$.

ЗАмечание 2. Отметим, что часть свойства (24), а именно то, что $D_{1,1}^{*} \subset B^{*}$, содержится в работе [1] (см. также [2]).

4. Докажем утверждение, дающее представление о геометрии множеств $B, B^{*}$ и $B_{0}$. Далее будем обозначать через $\mathbb{R}_{+}^{2}=\{(\xi, \eta): \xi>0, \eta>0\}$.

Теорема 3. Существует кривая $\sigma$ такая, что

1) $\sigma \subset \mathbb{R}_{+}^{2}$

2) кривую $\sigma$ можно задать параметрически $\sigma=\{(\xi, \eta): \xi=s(\eta), \eta>0\}$, причем функиия $s(\eta)$ не убъвает и удовлетворяет условию Липиица, т.е. если $\eta_{1}>\eta_{2}$, mo

$$
0 \leqslant s\left(\eta_{1}\right)-s\left(\eta_{2}\right) \leqslant \eta_{1}-\eta_{2}
$$

3) кривая $\sigma$ делит $\mathbb{R}_{+}^{2}$ на две связные части, одна из которых совпадает с В, а вторая принадлежит $B^{*}$, а именно:

$$
\begin{gathered}
B=\{(\xi, \eta): s(\eta)<\xi<\infty, \eta>0\}, \\
B^{*} \cup B_{0}=\{(\xi, \eta): 0<\xi \leqslant s(\eta), \eta>0\}, \quad B_{0} \subset \sigma .
\end{gathered}
$$


ДокАЗАТЕльСтво. Для произвольной точки $A=\left(\xi_{0}, \eta_{0}\right)$ обозначим через $l(A)$ луч, выходящий из точки $A$ параллельно оси $O \xi$ в направлении возрастания абсциссы $\xi$ : $l(A)=\left\{(\xi, \eta): \xi>\xi_{0}, \eta=\eta_{0}\right\}$.

Пусть $M$ - фиксированная точка оси $O \eta$ с положительной ординатой $\mu, M=(0, \mu)$, $\mu>0$.

Из (24) следует, что подмножество точек луча $l(M)$, принадлежаших множеству $B^{*}$, непусто и ограничено. Поэтому существует точка $C_{0}=\left(\alpha_{0}, \mu\right), \alpha_{0}>0$, такая, что для любого сколь угодно малого $\varepsilon$ найдется $\delta, \varepsilon>\delta \geqslant 0$, такое, что точка $C_{0 \delta}=\left(\alpha_{0}-\delta, \mu\right)$ принадлежит множеству $B^{*}$, а все точки луча $l\left(C_{0}\right)$ не принадлежат $B^{*}$. Отсюда в силу теоремы 1 следует, что все точки интервала $M C_{0 \delta}$ принадлежат $B^{*}$. Учитывая произвольность выбора $\varepsilon$, получаем

$$
M C_{0} \subset B^{*}, \quad l\left(C_{0}\right) \subset B \cup B_{0} .
$$

Аналогично, из (24) следует, что подмножество точек луча $l(M)$, принадлежащих множеству $B$, является непустым и их абсциссы ограничены снизу. Поэтому существует точка $C_{1}=\left(\alpha_{1}, \mu\right), \alpha_{1}>0$, такая, что для произвольного $\varepsilon$ найдется $\delta, \varepsilon>\delta \geqslant 0$, такое, что точка $C_{1 \delta}=\left(\alpha_{1}+\delta, \mu\right)$ принадлежит множеству $B$, а все точки интервала $M C_{1}$ принадлежат $B^{*} \cup B_{0}$. Из теоремы 1 следует, что все точки луча $l\left(C_{1 \delta}\right)$ принадлежат $B$. То есть

$$
l\left(C_{1}\right) \subset B, \quad M C_{1} \subset B^{*} \cup B_{0} .
$$

Докажем, что $C_{0}=C_{1}$. Допустим, что $C_{0} \neq C_{1}$. Тогда, очевидно, $\alpha_{1}>\alpha_{0}$ и из (27) и $(28)$ следует, что все точки интервала $C_{0} C_{1}$ принадлежат $B_{0}$. Поэтому если $C^{\prime}-$ произвольная точка интервала $C_{0} C_{1}$, то в силу теоремы 1 все точки интервала $C_{0} C^{\prime}$ принадлежат $B^{*}$, а интервал $C^{\prime} C_{1}$ лежит в $B$, что противоречит (27) и (28). Это означает, что точки $C_{0}$ и $C_{1}$ совпадают, и справедливы включения

$$
M C \subset B^{*}, \quad l(C) \subset B,
$$

где $C=C_{0}=C_{1}$.

Таким образом, для каждой точки $M=(0, \mu), \mu>0$, существует точка $C=C(\mu)$, $C \neq M$, которая делит луч $l(M)$ на интервал, лежащий в $B^{*}$, и луч, принадлежащий $B$. Объединение таких точек $C(\mu)$ для всех положительных $\mu$ обозначим через $\sigma$. Очевидно, что $\sigma$ представляет собой кривую, лежащую в $\mathbb{R}_{+}^{2}$, и ее можно задать параметрически $\sigma=\{(\xi, \eta): \xi=s(\eta), \eta>0\} ;$ при этом справедливы включения

$$
\{(\xi, \eta): s(\eta)<\xi<\infty, \eta>0\} \subset B, \quad\{(\xi, \eta): 0<\xi<s(\eta), \eta>0\} \subset B^{*} .
$$

Из этих включений, замечая, что $\mathbb{R}_{+}^{2}=B \cup B^{*} \cup B_{0}$, следует, что $B_{0} \subset \sigma$. Далее, пусть $\left(\alpha_{0}, \mu_{0}\right)$ - фиксированная точка из $\mathbb{R}_{+}^{2}$. Нетрудно заметить, что при фиксированном $x$ функция $E_{1 / \alpha}(x ; \mu)$ является непрерьвной функцией параметров $\alpha$ и $\mu$, по крайней мере, в некоторой окрестности точки $\left(\alpha_{0}, \mu_{0}\right)$. Поэтому, если $E_{1 / \alpha_{0}}\left(x_{0} ; \mu_{0}\right)<0$ для некоторого $x_{0}$, то, очевидно, $E_{1 / \alpha}\left(x_{0} ; \mu\right)<0$ для всех $(\alpha, \mu)$ из достаточной малой окрестности точки $\left(\alpha_{0}, \mu_{0}\right)$. То есть множество $B$ является открытым. Это означает, что точки кривой $\sigma$ не могут принадлежать множеству $B$, так как они являются для негограничными. Отсюда и из (29) следует (26). 
Докажем (25). Пусть $(\alpha, \mu) \in \sigma$, т.е. $\alpha=s(\mu)$. Тогда из (26) и теоремы 1 следует, что для любого $\delta>0$ верны включения $D_{\alpha-\delta, \mu} \subset B^{*}$ и $D_{\alpha+\delta, \mu} \subset B$. Поэтому в силу произвольности $\delta$ кривая $\sigma$ заключена между прямыми, проходяшими через точку $(\alpha, \mu)$, одна из которых параллельна оси $O \eta$, а вторая проходит через начало координат. То есть для произвольного $\varepsilon>0$ справедливы неравенства

$$
s(\mu) \leqslant s(\mu+\varepsilon) \leqslant \frac{\alpha}{\mu}(\mu+\varepsilon) .
$$

Отсюда, так как $\alpha=s(\mu)$, получаем

$$
0 \leqslant s(\mu+\varepsilon)-s(\mu) \leqslant \frac{s(\mu)}{\mu} \varepsilon .
$$

Из (23) следует, что $\mu \geqslant s(\mu)$, и мы приходим к $(25)$.

Выражаю искреннюю благодарность А. П. Солдатову и А. Ю. Попову за весьма полезные замечания, сделанные относительно этой работы.

\section{СПИСОК ЦИТИРОВАННОЙ ЛИТЕРАТУРЫ}

[1] Попов А. Ю., Седлецкий А. М. Распределение нулей функции Миттаг-Леффоера // Докл. РАН. 2003. Т. 390. № 2. С. 165-168.

[2] Седлецкий А. М. Неасимптотические свойства корней функции типа Миттаг-Лефффлера // Матем. заметки. 2004. Т. 75. № 3. С. 405-420.

[3] Нахушев А. М. Дробное исчисление и его применение. М.: Физматлит, 2003.

[4] Псху А. В. Интегральное преобразование с функцией Райта в ядре // Докл. Адыгской (Черкесской) Международной академии наук. 2002. Т. 6. № 1. С. 35-47.

[5] Станкович Б. О једној класи сингуларних интегралних једначина // Зборник радова САН. 43. Математички институт САН. 1955. Т. 4. С. 81-130.

[6] Wright E. M. The generalized Bessel function of order greater than one // Quart. J. Math. (Oxford). 1940. V. 11. P. 36-48.

[7] Псху А.В. Решение краевой задачи для уравнения с частными производными дробного порядка // Дифференц. уравнения. 2003. Т. 39. № 8. С. 1092-1099.

[8] Лаврентьев М.А., Шабат Б. В. Методы теории функций комплексного переменного. М.: Наука, 1987.

[9] Джрбашян М. М. Интегральные преобразования и представления функций в комплексной области. М.: Наука, 1966.

[10] Stanković B. On the function of E. M. Wright // Publs Inst. Math. Beograde. 1970. V. 10. № 24. P. 113-124.

Научно-исследовательский институт 